Musées, Patrimoine et Culture scientifiques et techniques

\title{
Le projet "Ingénieur en herbe"
}

\section{Jamila Al-Khatib}

URL : http://journals.openedition.org/ocim/1736

DOI : 10.4000/ocim. 1736

ISSN : 2108-646X

\section{Éditeur}

OCIM

Édition imprimée

Date de publication : 1 janvier 2017

Pagination : 26-32

ISSN : 0994-1908

Référence électronique

Jamila Al-Khatib, "Le projet "Ingénieur en herbe" », La Lettre de I'OCIM [En ligne], 169 | 2017, mis en ligne le 01 janvier 2018, consulté le 04 mai 2019. URL : http://journals.openedition.org/ocim/1736 DOl : 10.4000/ocim. 1736

Ce document a été généré automatiquement le 4 mai 2019.

Tous droits réservés 


\section{Le projet "Ingénieur en herbe"}

\section{Jamila Al-Khatib}

Des ingénieurs en herbe observent le diplôme reçu par leur classe.

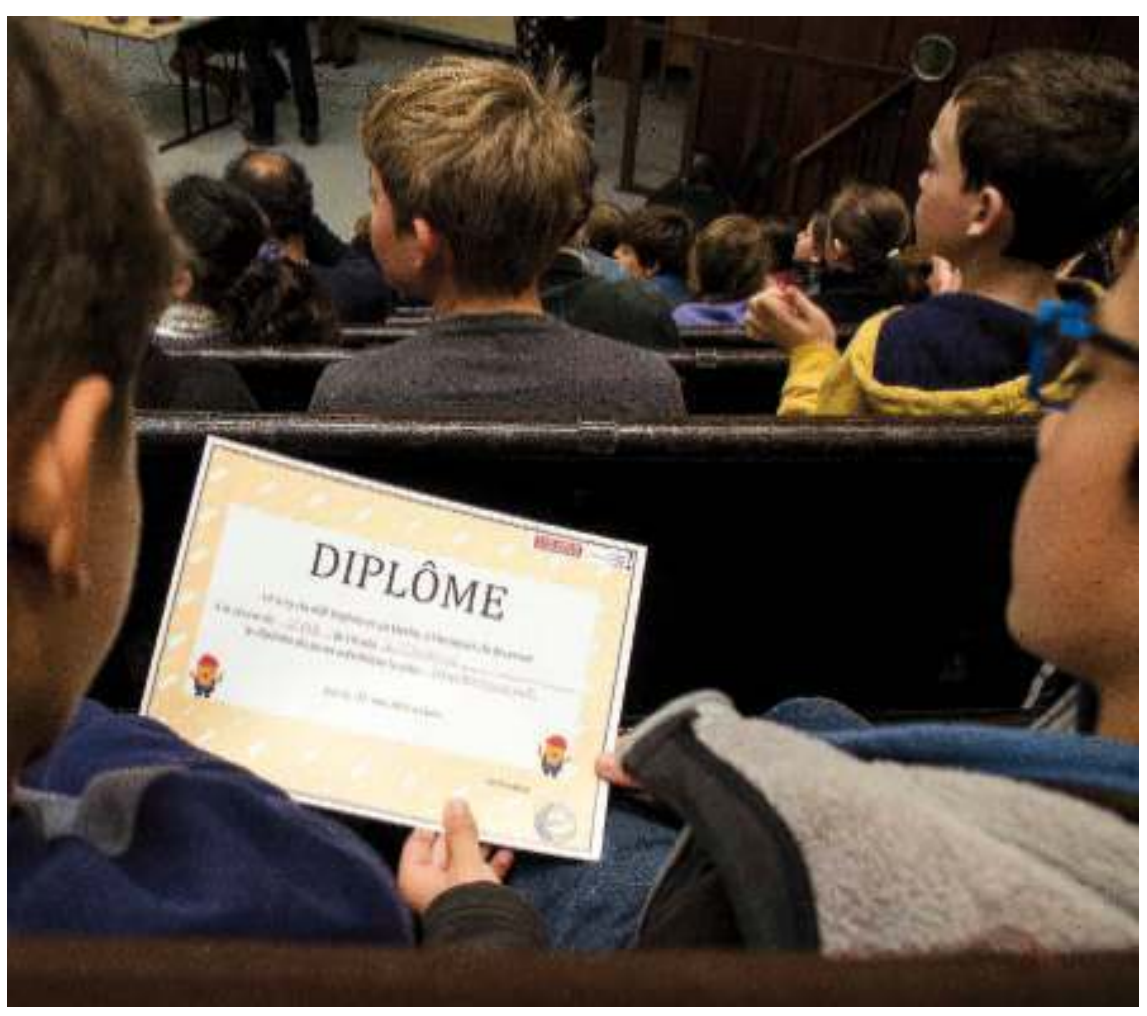

(c) Musée des Arts et Métiers

1 Le projet "Ingénieur en herbe" est à destination des groupes scolaires. En participant à ce projet, les élèves sont invités à se mettre dans la peau d'un ingénieur pour relever un défi. Les classes inscrites ont une année scolaire pour analyser un problème posé, mener des investigations, expérimenter, échanger avec des experts et réaliser un seul prototype pour l'ensemble de la classe, qui sera présenté à un jury d'ingénieurs et de professionnels 
de la médiation. Durant la première édition, soit l'année scolaire 2013/2014, les élèves ont conçu et réalisé un compteur mécanique : 15 classes ont participé, soit près de 430 élèves (10 classes de Paris et 5 classes des Hauts-de-Seine). Cette première édition nous a servi de test pour étudier la faisabilité du projet et de son déploiement. Pour la deuxième édition (année scolaire 2014-2015), le défi portait sur la conception et la réalisation d'un jouet mécanique : 55 classes ont participé, soit près de 1540 élèves (38 classes de Paris, 16 de l'académie de Versailles et 1 classe de l'académie de Créteil). Pour la troisième édition, les élèves ont conçu un pont mobile.

Dans le cadre de ce projet, le musée des Arts et Métiers apporte un soutien pédagogique à toutes les classes participantes. Les conseillères pédagogiques du ministère de l'Éducation nationale suivent ces classes et veillent à ce que ce projet s'inscrive dans les objectifs du programme scolaire, en faisant appel aux compétences suivantes :

3 - maîtrise des savoirs fondamentaux;

4 - utilisation des outils numériques permettant un travail collaboratif plus poussé entre pairs ;

5 - développement du parcours culturel des élèves ;

6 - découverte des métiers.

7 Ces éléments apportent à ce projet une envergure pluridisciplinaire, contribuant à l'accès à la culture scientifique et technique pour tous dans le cadre d'un partenariat permettant une liaison entre les écoles, les établissements et les institutions culturelles.

\section{Pourquoi le projet "Ingénieur en herbe" ?}

\section{Présentation du musée des Arts et Métiers}

Le musée des Arts et Métiers est un service du Conservatoire national des arts et métiers, grand établissement public à caractère scientifique, culturel et professionnel. Ce musée s'est constitué à partir des collections historiques préservées par le Conservatoire dès sa création en 1794. Comportant des collections anciennes, comme le Laboratoire de Lavoisier ou l'avion de Louis Blériot ayant fait la traversée de la Manche, il possède également des pièces remarquables mettant en évidence le geste de l'ingénieur et montrant les évolutions apportées à une famille d'objets techniques. Musée national, il possède un savoir-faire en matière de médiation, dans le domaine de la diffusion de la culture scientifique et technique, qui fait référence. Cet établissement propose des activités pédagogiques à destination du public en général et des groupes scolaires et des étudiants en particulier. Chaque année, près de 40000 scolaires visitent le musée, en participant à des visites guidées, des ateliers pédagogiques ou en utilisant les ressources pédagogiques librement téléchargeables à partir du site Internet du musée. 
Des moments capturés lors de la restitution.

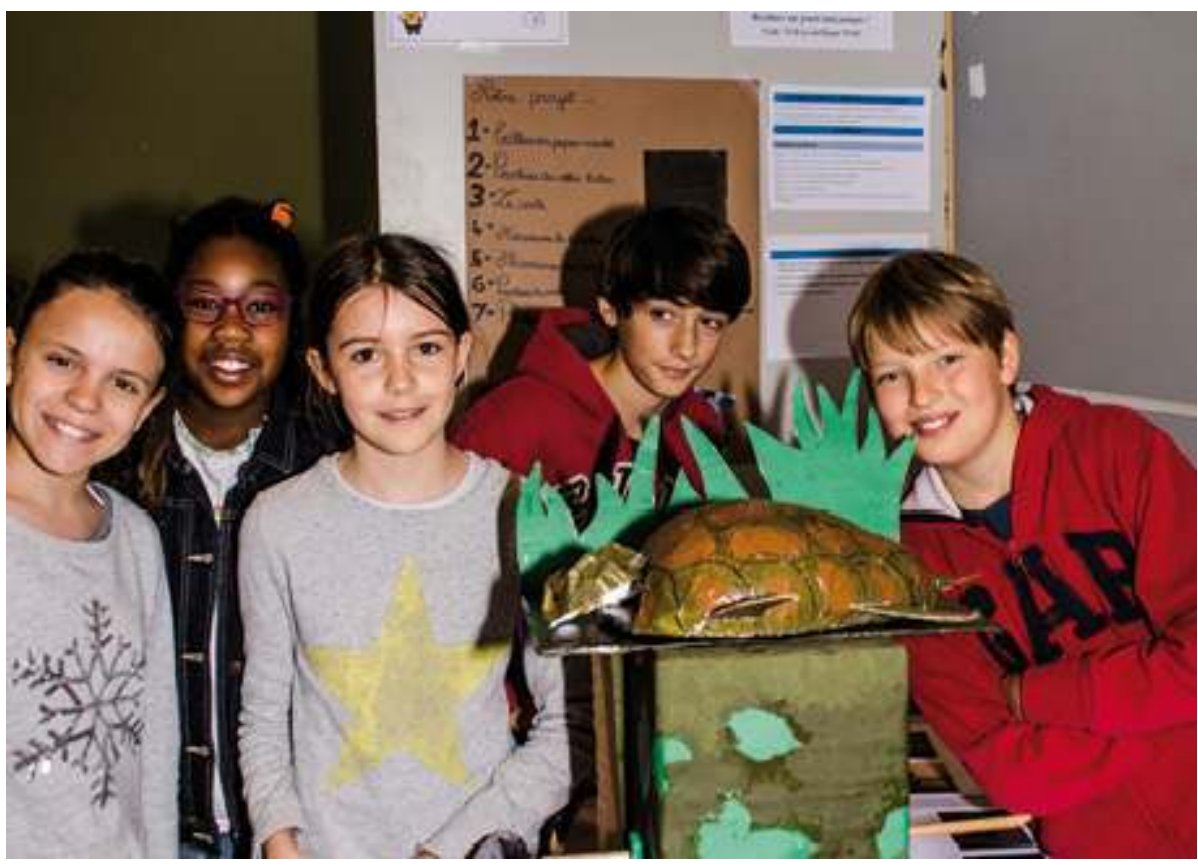

(c) Musée des Arts et Métiers

\section{ENGINEER, Une opération pédagogique innovante}

Dans un rapport parlementaire portant sur l'enseignement scolaire (octobre 2013), la députée Julie Sommaruga affichait la volonté de développer la culture scientifique pour stimuler le potentiel économique français, l'innovation, la compétitivité et la recherche. Les évaluations menées par le ministère de l'Éducation nationale avaient montré qu'à résultats comparables, les filles souffraient d'une inégalité d'orientation et s'autocensuraient par rapport aux carrières d'ingénieurs, souvent perçues comme n'étant pas féminines. Une partie du rapport présentait des pistes pour améliorer l'enseignement des sciences en renforçant l'interdisciplinarité, la formation professionnelle et le lien école-collège.

D’octobre 2011 à septembre 2014, le musée des Arts et Métiers a participé au projet européen ENGINEER (BrEaking New Ground IN the SciencE Education Realm), dont l'objectif était de faire découvrir le métier et la démarche de l'ingénieur par le biais d'activités pédagogiques. Ce projet a rassemblé 26 partenaires issus de 10 pays membres (Allemagne, Angleterre, Danemark, France, Grèce, Israël, Italie, Pays-Bas, République Tchèque et Suède). Durant trois ans, des musées de sciences et de techniques ont collaboré avec des écoles primaires, pour concevoir des animations pédagogiques destinées à des élèves âgés de 9 à 11 ans. Chaque binôme (musée/école) a développé un dossier pédagogique contenant des activités à destination des élèves, des formateurs d'enseignants et des acteurs de la diffusion de la culture scientifique et technique. Ces activités ont été conçues en suivant la démarche pédagogique EiE, Engineering is Elementary mise au point par le musée de Boston, pour encourager les enfants, y compris ceux issus des zones prioritaires, à se projeter comme de futurs ingénieurs. 
11 Des activités ont été conçues, animées et évaluées dans les champs d'ingénierie suivants : le biomédical (les principes de la respiration), la géotechnique (les propriétés du sol), l'acoustique (la propagation du son), l'électricité (la fabrication d'un aspirateur), l'environnement (la culture des plantes), les matériaux (les propriétés thermiques des tissus), l'océan (le principe des plateformes), l'aéronautique (la fabrication d'un avion), l'industrie (la notion d'équilibre avec les balances), la mécanique (la réalisation d'un jouet et d'un compteur mécaniques).

12 En septembre 2014, à la fin du projet ENGINEER, plus de 100 enseignants du 1er degré et 450 élèves avaient bénéficié de ces activités, en France. À l'échelle de l'ensemble des partenaires du projet, près de 660 écoles, 1000 enseignants et 27000 élèves ont participé à ces activités.

13 Fort de cette expérience, en septembre 2013, le musée des Arts et Métiers a lancé une nouvelle opération pédagogique intitulée "Ingénieur en herbe" à destination des groupes scolaires du cycle 3 , soit les élèves âgés de 9 à 11 ans.

\section{Pourquoi s'intéresser aux sciences de l'ingénieur?}

14 Nous vivons exclusivement dans un monde que nous modifions au gré de nos besoins avec l'aide des ingénieurs. En effet, de par sa définition officielle, l'ingénieur est celui qui " mène à bien une opération industrielle ou un projet technique mettant en ceuvre des ressources humaines, des moyens techniques et financiers et dont les résultats sont soumis aux réalités socioéconomiques".

Cependant, l'étude de cet aspect ne relève pas encore des programmes scolaires, essentiellement axés sur l'étude de concepts scientifiques. Si les jeunes ont une représentation d'un scientifique, ils ont beaucoup plus de mal à se représenter ce qu'est un ingénieur. Pour eux, les ingénieurs construisent des immeubles, réparent des voitures ou des ordinateurs ou conduisent des trains. Peu ont conscience du fait que les ingénieurs conçoivent toutes les technologies qui nous entourent.

Nous pensons qu'à court terme, étudier les sciences de l'ingénieur est l'occasion de développer un projet pluridisciplinaire, rendant accessibles aux élèves des disciplines pouvant être difficiles, telles les mathématiques, le français ou la science. Mais, cette étude promeut également des facultés cognitives utiles, telles que la pensée critique, la collaboration, la communication, la créativité, la souplesse, la persévérance et l'apprentissage à partir des échecs.

17 À long terme, pratiquer les sciences de l'ingénieur permet d'offrir une égalité des chances pour chaque élève de briguer une carrière d'ingénieur.

18 Nous sommes donc persuadés que l'enseignement de la démarche de l'ingénieur devrait faire partie du curriculum scolaire dès le niveau primaire, afin que les futurs citoyens puissent définir et résoudre les défis techniques de demain, avant d'en être dépassés. Participer au projet "Ingénieur en herbe" y contribue. De par ses collections, son expérience en médiation scientifique et culturelle et son réseau, le musée des Arts et Métiers est un acteur incontournable pour coordonner le projet pédagogique "Ingénieur en herbe", en collaboration avec des partenaires scolaires et culturels. 
Un schéma technique décrivant une réalisation.

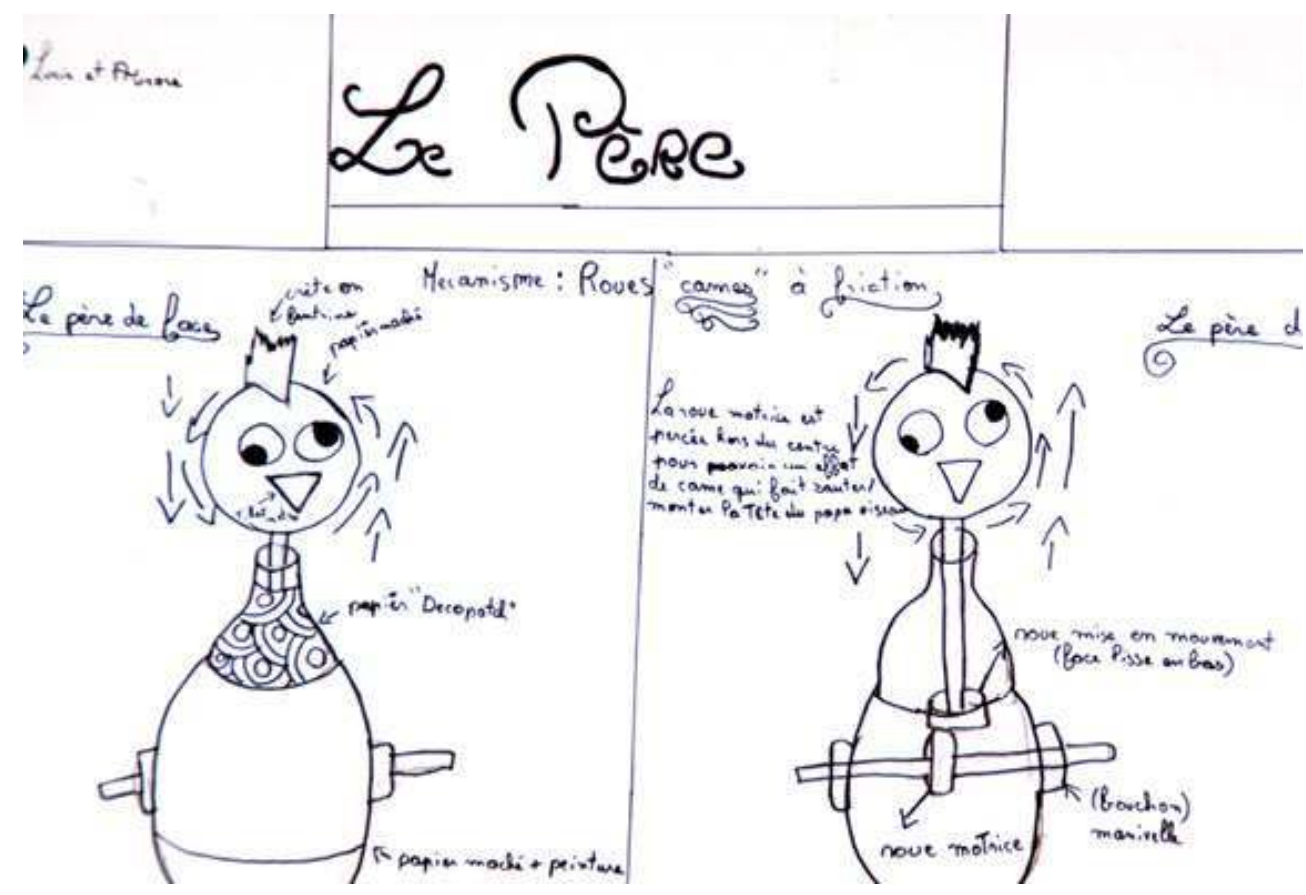

(c) Musée des Arts et Métiers

\section{Intervenants du projet}

La réussite de ce projet réside essentiellement dans la collaboration et l'implication de plusieurs acteurs. Certains constituent le comité de pilotage et s'impliquent directement dans la conception et l'animation du projet et d'autres interviennent plus ponctuellement. Nous avons basé les principes de ce projet sur l'interaction, vue comme une source d'apprentissage et de développement professionnel, suivant les théories socioconstructivistes et sociocognitives de l'apprentissage. Ces derniers se traduisent au niveau individuel, collectif ou organisationnel, s'ils contribuent à des pratiques en lien avec le projet de l'établissement.

\section{Les partenaires}

Dès la première édition, le projet a été conçu conjointement avec le ministère de l'Éducation nationale, dont quatre conseillères pédagogiques de l'académie de Paris et deux de Versailles. Des partenaires industriels (comme l'UIMM, Union des Industries et des Métiers de la Métallurgie) et universitaires (Université de Saint-Quentin en Yvelines, Versailles) ont été approchés. Si leur participation a été plus transversale la première année, elle a été plus active la seconde année.

Pour la seconde édition, de nouveaux partenaires ont rejoint le projet : trois conseillères pédagogiques de Paris, deux de Créteil, deux professeurs de collège, les élèves d'un lycée professionnel, la responsable de l'action éducative du musée des Arts décoratifs, les médiateurs de la Maison des sciences (le centre-pilote La main à la pâte de ChâtenayMalabry), la cheffe de projet de la culture scientifique de la mairie d'Ivry-sur-Seine. 
L'ensemble de ces partenaires a constitué un réseau fiable de communication envers les enseignants et les institutionnels pour assurer la pérennité du projet. Aujourd'hui, pour assurer sa faisabilité, le musée des Arts et Métiers apporte à toutes les classes participantes un soutien pédagogique et finance lui-même ses activités (formation des enseignants et visites guidées pour tous les groupes scolaires, organisation des journées de restitution, envoi du matériel, opération de communication...), le musée des Arts décoratifs apporte des ressources pédagogiques et finance lui-même ses activités (formation des enseignants et des ateliers pour quelques groupes scolaires), les conseillères pédagogiques apportent un soutien pédagogique aux enseignants participants et mettent à leur disposition des ressources pédagogiques et les enseignants s'engagent à mener le projet jusqu'au bout.

Les liens nécessaires avec le programme scolaire sont assurés tout au long du projet, garantissant aux enseignants que la participation à ce projet s'inscrit parfaitement dans les exigences du curriculum scolaire. Cette collaboration a été l'occasion de souligner la complémentarité entre les métiers de l'enseignement et ceux de la médiation, afin de concevoir un projet interdisciplinaire, didactique et pérenne.

Un exemple de réalisation de jouet mécanique

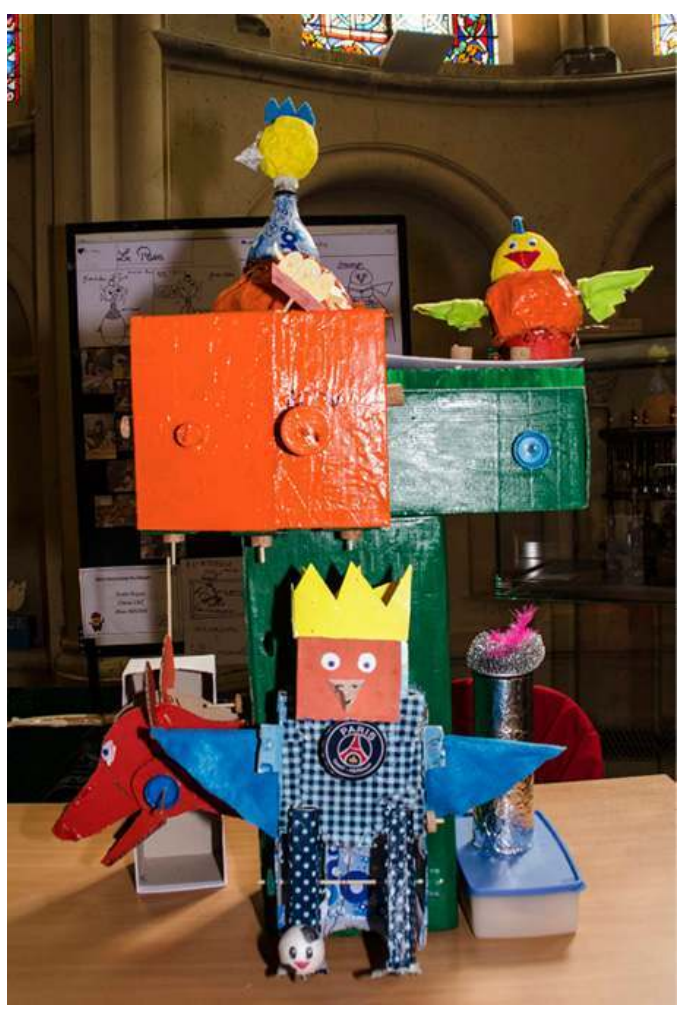

(C) Musée des Arts et Métiers

\section{Les bénéficiaires}

Pour la première édition, nous avons décidé de proposer le projet uniquement aux élèves de niveau CM1-CM2 pour s'inscrire dans la continuité du projet ENGINEER. Pour la seconde édition, le projet a été étendu aux élèves de CE2 et aux collèges. 

permettant ainsi de se confronter au défi et d'y voir clairement les liens avec le programme scolaire. Cette formation se déroule pendant trois ou six heures, en fonction du cahier des charges de formation des enseignants établi par les académies. Ainsi, les relations entre les trois intervenants (l'enseignant, la conseillère pédagogique et le médiateur) sont renforcées. Il nous a semblé important que les enseignants puissent, dès le démarrage du projet, identifier leurs interlocuteurs, ainsi que les aides dont ils peuvent bénéficier. 
Les ingénieurs en herbe présentent leur prototype au jury.

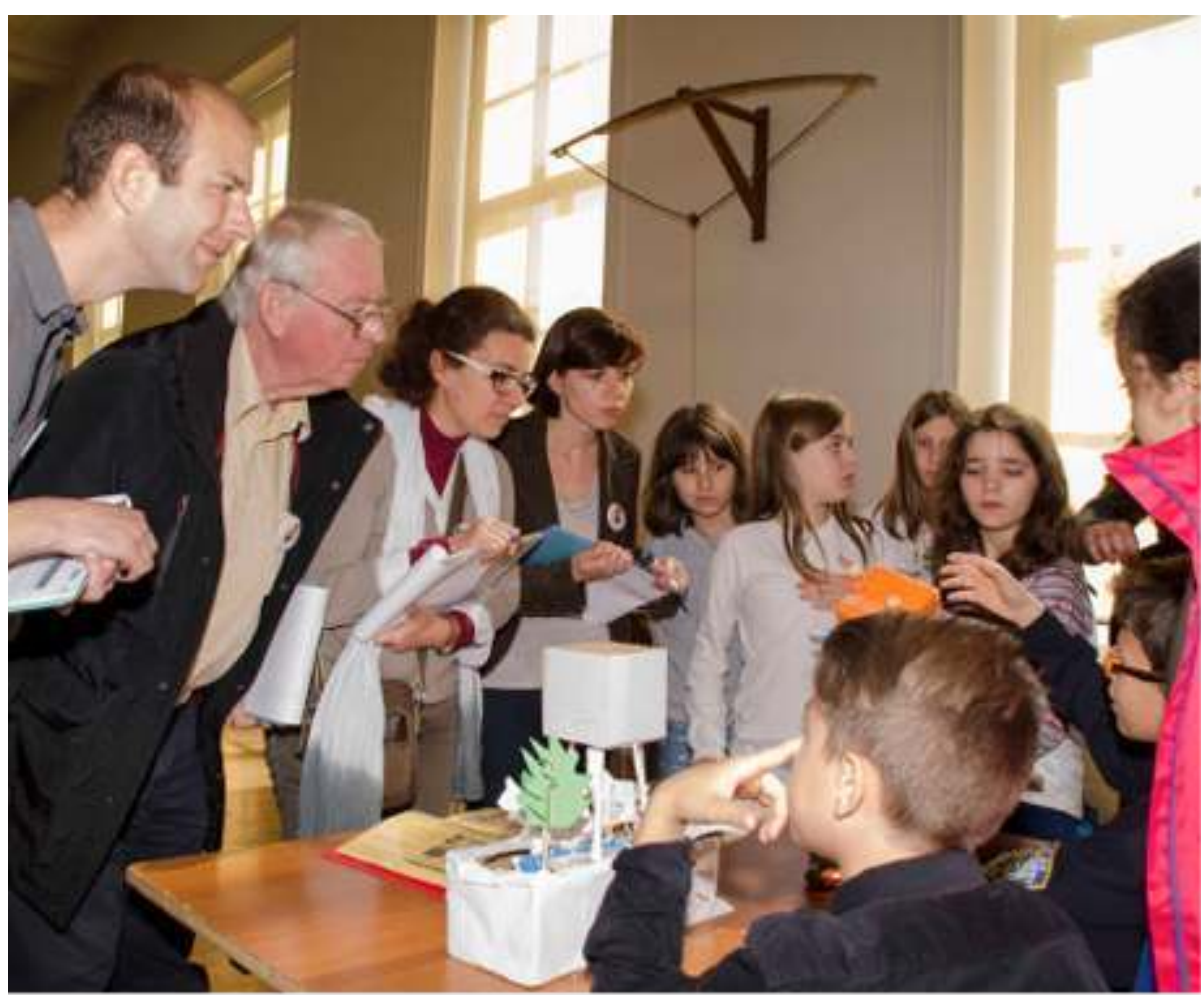

(C) Musée des Arts et Métiers

\section{Étape 3 : inscription des classes}

En classe, l'enseignant étudie l'appel d'offre avec ses élèves, répondant ainsi aux exigences du programme scolaire : apprendre à utiliser les nouvelles technologies et pratiquer la langue française. Cette étape se termine par la rédaction et l'envoi d'une seule lettre de motivation pour l'ensemble de la classe. C'est le début du travail collaboratif. Les enseignants peuvent mobiliser plusieurs disciplines scolaires, telles les arts plastiques pour réaliser une "belle" lettre et/ou le français pour analyser avec leurs élèves les exigences administratives d'une lettre officielle. L'objectif de cette étape est de montrer que, contrairement à ce que peuvent penser les élèves, un ingénieur doit être capable de manier l'écrit, comme les sciences et/ou les mathématiques. Certains enseignants saisissent cette occasion pour former de futurs citoyens à écrire et envoyer des lettres officielles.

\section{Étape 4 : validation de la candidature}

Dès réception de la lettre de motivation, le musée crée un compte électronique pour la classe lui permettant de poster des commentaires sur un blog conçu pour ce projet. L'objectif est de constituer une communauté qui partage ses trucs et astuces, avant de se retrouver le jour de la restitution. Consulter et poster des commentaires sur cette plateforme relève également des compétences à acquérir dans le cadre du programme scolaire. Puis le musée envoie à chaque classe le matériel imposé. Il s'agit là d'un clin d'œil à l'ingénieur qui répond à un appel d'offre et doit respecter un budget. Nous avons 
volontairement choisi du matériel simple (carton, feutres, pailles, pâte à modeler...), pour insister davantage sur l'importance de la démarche d'investigation des élèves que sur le produit fini. Les élèves peuvent cependant y rajouter du matériel recyclé.

\section{Étape 5 : phase de résolution du défi}

L'enseignant et ses élèves sont suivis par une conseillère pédagogique et par un médiateur du musée, au besoin. Le musée assure un accès à ses collections, de façon à permettre aux élèves de puiser des idées dans son patrimoine scientifique et technique, et y mener des investigations fructueuses. Ainsi, chaque classe est accueillie pour une visite guidée, durant laquelle le médiateur donne les clés de lecture muséographique pour analyser les objets de collection présentés et en retirer des éléments de connaissance pour la résolution du défi, développant ainsi chez les élèves une culture artistique, scientifique et technique. Cette année, le renforcement du plan Vigipirate a empêché un certain nombre de classes de se déplacer au musée. Les médiateurs du musée ont alors adapté la visite guidée en une présentation/intervention faite en classe.

\section{Étape 6 : présentation du prototype}

L'opération se conclut sur un temps de restitution, pendant lequel les ingénieurs en herbe présentent leurs réalisations à un jury d'experts constitués d'ingénieurs et de professionnels de la pédagogie. C'est l'occasion pour les élèves de raconter leur travail et de défendre leurs choix. Un diplôme est ensuite remis à chaque classe participante. Pour la seconde édition, le jury est constitué des personnalitées suivantes : des conseillers pédagogiques, une doctorante, des responsables du musée des Arts décoratifs, une représentante d'une start-up, des responsables du musée des Arts et Métiers, des étudiants du lycée professionnel de carrosserie de Sarcelles, des ingénieurs, des enseignants de physique-chimie.

Nous avons conçu ce projet pour permettre aux élèves de devenir des citoyens avisés, développant ainsi leur goût de l'effort et leur sens critique. En effet, le contenu du projet est conçu de façon à stimuler chez l'élève le questionnement et une capacité à construire, structurer et matérialiser un projet. À chaque étape du projet, il doit interroger son prototype, y compris durant sa visite au musée qui vient nourrir sa réflexion. Nous avons aussi choisi de montrer que tout apprentissage est plus riche s'il se pratique dans des milieux et contextes différents (l'école, les institutions culturelles et les expériences personnelles), permettant ainsi de poursuivre un apprentissage en dehors du milieu scolaire traditionnel. 


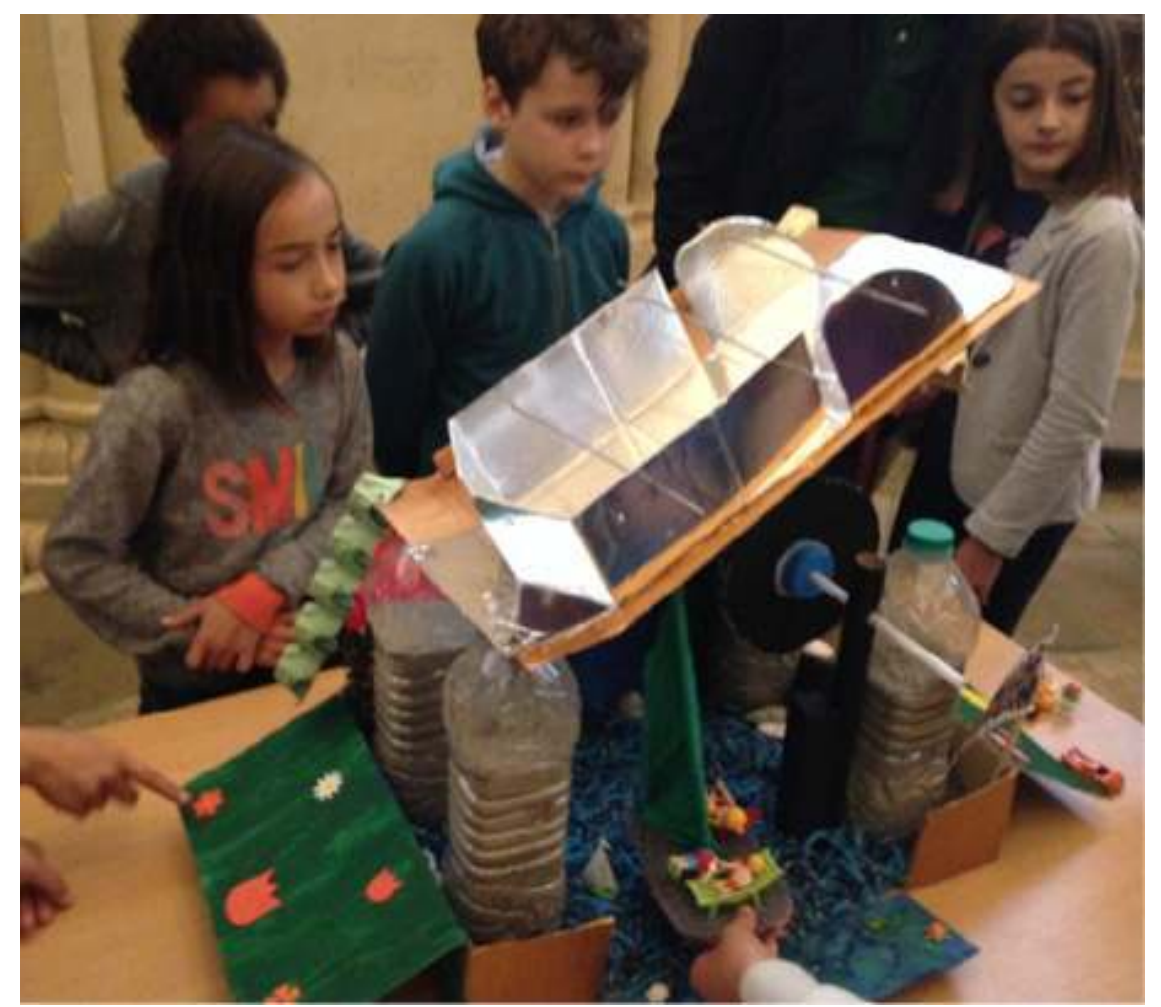

() Musée des Arts et Métiers

\section{Retour des évaluations}

Pour étudier l'efficacité de cette opération, nous avons mené une enquête, sous forme d'un questionnaire à choix multiple, auprès des élèves ayant participé au projet. L'objectif était de mieux connaître les ingénieurs en herbe et savoir comment ils ont perçu cette expérience, afin de réajuster le contenu du projet, pour mieux répondre à leurs attentes en fonction de nos objectifs pédagogiques. Nous avions placé ce projet dans le cadre d'une démarche pédagogique qui a fait ses preuves. Sa conception s'appuie sur quatre piliers favorisant les capacités suivantes (Surlemont et Kearney 2009) :

- un apprentissage responsabilisant : les participants contrôlent le processus d'apprentissage et sont responsables de leurs choix dans un contexte sécurisé, où l'incidence du risque est négligeable ;

37 - un apprentissage en direct : les participants font des expériences et apprennent au cours de l'action, mais aussi après l'action ;

- un apprentissage coopératif : les expériences se font en équipe et de nombreux échanges et débriefings sont faits en groupes conviviaux ;

- un apprentissage réflexif : les participants sont sollicités pour réfléchir à partir de leurs expériences antérieures et celles vécues en cours de formation.

Nous avons choisi de présenter les résultats sous forme de réponse à nos questions. 


\section{Quelle est la typologie des ingénieurs en herbe?}

41 Pour la première édition, nous avons accueilli 10 classes, comprenant $56 \%$ de filles et 44 $\%$ de garçons, âgés en moyenne de 10 ans. Pour l'édition 2, nous avons accueilli 55 classes, comprenant $54 \%$ de filles et $46 \%$ de garçons, âgés en moyenne de 10 ans. Bien que l'on tende à penser que les projets scientifiques intéressent davantage les garçons, nous constatons que les participants sont pour moitié des filles, vu la constitution des groupes scolaires. Nous aurons donc le point de vue des filles et des garçons sur notre projet.

\section{Les ingénieurs en herbe participent-ils souvent à des projets ?}

On constate que les classes accueillies ont l'habitude de participer à des projets pédagogiques $(82 \%)$. Cependant $41 \%$ seulement ont déjà participé à des projets scientifiques. Parmi ces classes, $65 \%$ des élèves étaient déjà venus visiter le musée des Arts et Métiers avant le projet, soit dans un contexte scolaire, soit dans un contexte familial. Par ailleurs, 72 \% des élèves ont beaucoup apprécié l'idée de devoir résoudre un défi pour un musée.

\section{Comment les ingénieurs en herbe ont-ils jugé le déroulé du projet ?}

Pour la première édition, $97 \%$ des élèves ont aimé découvrir le défi à résoudre ; $74 \%$ ont apprécié de découvrir le matériel envoyé ; seulement $41 \%$ des élèves ont aimé écrire la lettre de motivation. La majorité (64\%) des participants ont préféré la partie "émission des hypothèses et tests de ces dernières". Seuls $38 \%$ des élèves ont assuré que la visite du musée des Arts et Métiers leur a donné des idées pour le défi : il s'agissait des élèves venus visiter le musée au début du projet. Nous avons modifié le contenu de la visite dès la seconde édition pour répondre aux attentes des groupes venant à mi-parcours. $71 \%$ ont apprécié la visite sous forme de "jeu", dont l'objectif consistait en la présentation d'un objet de collection, par les élèves eux-mêmes pour leurs camarades. Le seul échec de cette première édition était le fonctionnement du blog dédié au projet. Sur la question "As-tu aimé consulter et publier sur un blog ?" : $49 \%$ ont répondu négativement et $37 \%$ n'ont pas donné de réponse.

Pour la deuxième édition, le défi était de concevoir un jouet mû de façon mécanique, $96 \%$ des élèves ont aimé découvrir le défi à résoudre, $63 \%$ ont apprécié découvrir le matériel envoyé, $53 \%$ ont aimé écrire la lettre de motivation. La majorité des élèves ont apprécié le fait de résoudre un défi pour un musée ainsi qu'émettre des hypothèses et les tester. La visite sous forme de jeu a encore été un franc succès auprès des élèves. Le blog dédié au projet a davantage été mis en valeur, beaucoup de professeurs publiaient régulièrement des articles sur l'avancée de leur projet, illustrés de photographies, croquis...

Nous envisageons de modifier le questionnaire pour cette troisième édition, afin de l'axer sur des questions relatives au regard que portent ces élèves sur les musées.

\section{Comment les ingénieurs en herbe ont-ils jugé la restitution du projet?}

Lors de la restitution du projet au mois de mai, les élèves ont eu l'occasion d'échanger avec des experts (ingénieurs, professionnels de la médiation et autres ingénieurs en herbe). $70 \%$ des élèves ont estimé ce moment de présentation de leurs travaux, instructif 
et intéressant, et $68 \%$ des élèves ont trouvé très bonne, l'idée de se rencontrer entre eux et de découvrir que chaque classe a réalisé un prototype différent.

Lors de la deuxième édition, la majorité des élèves $(82 \%)$ ont beaucoup aimé participer à cette restitution. Par ailleurs, tout comme l'année précédente la majorité des élèves ont apprécié d'être réunis afin de découvrir et comparer leurs différentes réalisations.

\section{Les perspectives...}

48 Ce projet a permis d'offrir aux enfants participants à ce projet, une occasion de rencontres, d'échanges et de partage dans un lieu attrayant : le musée. Si nous avons consolidé la relation école-musée à travers ce projet ancré dans la réalité des élèves, nous avons aussi développé une expertise sur les projets éducatifs faisables et transférables. Nous envisageons les progressions suivantes :

49 - élargir le niveau scolaire des participants jusqu'aux deux premiers niveaux du collège (6 e et 5e) pour s'appuyer sur l'interaction directe entre les classes de primaire et de collège ;

50 - renforcer notre collaboration avec le Cnam Centre, qui déploie aussi ce projet en collaboration avec la Maison des Sciences d'Orléans ;

51 - développer un partenariat avec le Pavillon de Manse (Chantilly) pour étudier la transférabilité du projet.

Nous avons dès à présent lancé une troisième édition durant laquelle les ingénieurs en herbe devaient concevoir et réaliser un pont mobile de façon mécanique. Et depuis septembre 2016, nous travaillons déjà à la 4e édition, qui portera sur la thématique des transports. Les élèves auront ainsi le loisir d'imaginer un transport permettant à la mascotte du projet de rejoindre sa cabane.

Description de la démarche EiE (Engineering is Elementary)

L'objectif est de résoudre un problème technique en suivant les cinq étapes de raisonnement suivantes :

- se questionner pour cibler les objectifs du problème ;

- imaginer des propositions ;

- planifier ces propositions en sélectionnant le matériel et les outils nécessaires ;

- créer/réaliser le(s) prototype(s);

- porter un regard critique sur le(s) prototype(s) réalisés(s) pour les améliorer.

L'expérience montre que ces cinq étapes se positionnent sur un cycle de démarche cognitive. En effet, arrivé à l'étape "Améliorer", le participant doit s'assurer que les améliorations proposées répondent aux objectifs du problème et reprend le cycle. Ces cinq étapes ont été schématisées par les pédagogues du musée des sciences de Boston. Pour en savoir plus, la démarche est décrite sur leur site : www.eie.org. 


\section{BIBLIOGRAPHIE}

Bourgeois, E. et Chapelle, G. Apprendre et faire apprendre. Paris : PUF, 2011.

Surlemont, B. et Kearney, P, Pédagogie et esprit d'entreprendre. De Boeck Supérieur, 2009.

Jacomy, B. Une histoire des techniques. Seuil, 1990.

https://ingenieurenherbedefi.wordpress.com

Description du projet dans un article paru dans le journal municipal de Bagneux (magazine de juillet/août) : https://nassimaouail.wordpress.com/2015/05/28/un-crocodile-sinvite-dans-uneclasse-de-cm1/.

\section{RÉSUMÉS}

Mené dans le cadre d'un partenariat entre l'Éducation nationale, l'université, des industriels et le musée des Arts et Métiers, le projet "Ingénieur en herbe" s'est fixé comme principal objectif de donner au public scolaire le goût des sciences et des technologies en favorisant l'accès à la culture scientifique et technique: l'une des responsables dresse un premier bilan de cette opération et envisage les perspectives de développement du concept.

\section{INDEX}

Mots-clés : médiation, scolaire

\section{AUTEUR}

\section{JAMILA AL-KHATIB}

Responsable du département Médiation au musée des Arts et Métiers et chargée du projet "Ingénieur en herbe" jamila.al_khatib@cnam.fr 